\title{
The effects of a reduced-sodium, high-potassium salt substitute on food taste and acceptability in rural northern China
}

\author{
Nicole $\mathrm{Li}^{1 *}$, John Prescott ${ }^{2}$, Yangfeng $\mathrm{Wu}^{3,4,5}$, Federica Barzi ${ }^{1}$, Xuequn $\mathrm{Yu}^{3,4}$, Liancheng Zhao ${ }^{3}$, \\ Bruce Neal ${ }^{1}$ for the China Salt Substitute Study Collaborative Group ${ }^{4} \dagger$ \\ ${ }^{1}$ The George Institute for International Health, University of Sydney, PO Box M201, Camperdown, Sydney, NSW 2050, Australia \\ ${ }^{2}$ School of Psychology, University of Newcastle, Ourimbah, NSW, Australia \\ ${ }^{3}$ Cardiovascular Institute and Fuwai Hospital, Beijing, People's Republic of China \\ ${ }^{4}$ The George Institute China, Beijing, People's Republic of China \\ ${ }^{5}$ Peking University Health Science Centre, Beijing, People's Republic of China
}

(Received 25 January 2008 - Revised 5 June 2008 - Accepted 28 June 2008 - First published online 19 August 2008)

A potassium chloride-containing salt substitute lowers blood pressure levels, but its overall acceptability has been of concern due to its potential adverse effects on food taste. In a large-scale, blinded randomised trial evaluating the comparative effects of a salt substitute (65\% sodium chloride, $25 \%$ potassium chloride and $10 \%$ magnesium sulphate) and a normal salt (100\% sodium chloride) on blood pressure, we collected data on the saltiness, flavour and overall acceptability of food. We performed this at baseline, 1, 6 and 12 months post-randomisation using $100 \mathrm{~mm}$ visual analogue scales for assessments of both home-cooked foods and a standard salty soup. The mean age of the 608 participants from rural northern China was 60 years and $56 \%$ of them were females. In the primary analyses, the changes in the saltiness, flavour and overall acceptability of both home-cooked foods and a standard salty soup were not different between the randomised groups (all $P>0.08$ ). In the secondary analyses, weighting each of the data points according to the lengths of the respective follow-up intervals, the flavour of both home-cooked foods (mean difference $=-1.8 \mathrm{~mm}, P=0.045$ ) and a standard salty soup (mean difference $=-1.9 \mathrm{~mm}, P=0.03$ ) was slightly weaker in the salt substitute group. We conclude that salt substitution is both an effective and an acceptable means of blood pressure control. Possible small differences in flavour did not importantly deter the use of the salt substitute in this study group, although the acceptability of the salt substitute by a more general population group would need to be confirmed.

Randomised-controlled trials: Salt: Sodium: Potassium: Taste: China: Blood pressure

Worldwide it is estimated that about two-thirds of the cerebrovascular disease burden and one-half of the coronary disease burden are attributable to non-optimal blood pressure levels ${ }^{(1)}$. Blood pressure lowering based on dietary salt reduction has been identified as a potentially highly cost-effective means of CVD prevention ${ }^{(2)}$. Salt substitutes with reduced sodium and added potassium lower blood pressure ${ }^{(3,4)}$, but have been considered to have limited applicability in economically developed countries where most dietary salt comes from processed foods and cannot easily be replaced with a salt substitute at home. This is not the case in developing regions such as rural northern China where salt intake is high ${ }^{(5)}$, most dietary salt comes from foods cooked at home and the blood pressure reductions achievable with a salt substitute may be large ${ }^{(6)}$. The China Salt Substitute Study, which tested the effects of a commercially available salt substitute ${ }^{(7)}$ on blood pressure in high-risk rural northern Chinese populations, showed that a significant and sustained reduction in systolic blood pressure could be achieved ${ }^{(8)}$. To support the widespread uptake of a salt substitute in the region, it is also necessary to have clear evidence about the effects of the intervention on food flavour since such sensory factors are a crucial determinant of food choices ${ }^{(9,10)}$. In the case of a salt substitute containing potassium, bitterness may be a barrier to food acceptability, at least initially ${ }^{(11)}$.

The China Salt Substitute Study included a specific assessment of the impact of the salt substitute on saltiness, flavour and overall acceptability of food flavour, and the results are reported here. Since a reduction in dietary sodium consumption might be expected to produce preferences for lower levels of salt in other foods ${ }^{(12)}$, assessments were made not only of the usual diet, but also of a standard salty soup administered at intervals throughout the study.

\section{Methods}

The design and results of the China Salt Substitute Study have been published ${ }^{(8)}$. In brief, the study was a double-blind, randomised, controlled trial that demonstrated clear blood pressure-lowering effects of a reduced-sodium, high-potassium salt

* Corresponding author: Dr Nicole Li, fax +61 29993 4502, email nli@george.org.au

$\dagger$ Members listed in acknowledgements. 
substitute among 608 individuals, at elevated risk of CVD, living in rural China. The study was carried out between May 2004 and August 2005 at thirty-nine sites distributed between six regional coordinating centres in northern China (Heilongjiang, Tianjin, Liaoning, Shanxi and two centres near Beijing). The trial received ethics approvals from the University of Sydney, Sydney, Australia and the $\mathrm{Fu}$ Wai Hospital, Beijing, People's Republic of China, and all the participants provided informed consent.

\section{Participants}

Individuals were eligible for the study if they had a high risk of future vascular disease based on a doctor's diagnosis of any of the following: (1) coronary, cerebral or peripheral vascular disease; (2) diabetes and aged 55 years or older; (3) a systolic blood pressure $>160 \mathrm{mmHg}$. In addition, all the participants were required to have an estimated daily sodium intake of $260 \mathrm{mmol} / \mathrm{d}$ or more and an expectation that at least half of the dietary salt could be replaced with the study salt substitute/salt. This was estimated through an interview with the patient or the individual responsible for daily food preparation (if this was not the patient) using a structured questionnaire at the baseline visit. High-risk individuals were selected for the study as they are the population group likely to attain more benefit from blood pressure lowering. Finally, the participants were required to have no established clear indication for, or contraindication to, the use of the study salt substitute, such as the use of a potassium-sparing medication or significant renal impairment.

\section{Randomisation}

Randomisation (assignment of the participants to intervention or control as if by the toss of a coin) was done using a central computerised randomisation service accessed by centre physicians via the study website with a back-up phone and fax service. A computer program performed the randomisation with stratification within each centre by baseline systolic blood pressure (in three strata) and present use of antihypertensive therapy. The service was maintained by the Clinical Trials Research Unit at the University of Auckland, New Zealand. The randomisation service provided a unique number for each individual corresponding to a treatment pack held at the centre. Treatment allocation was blinded to study investigators, participants and centre physicians until the study database was locked.

\section{Study treatment}

Study treatment comprised a salt substitute for all the participants who entered the run-in period and either a salt substitute or a normal salt for those participants who were randomised. The salt substitute was $65 \%$ sodium chloride, $25 \%$ potassium chloride and $10 \%$ magnesium sulphate. This formulation was commercially available in China and had been evaluated previously $^{(7)}$. The normal salt was $100 \%$ sodium chloride. Randomised treatment was delivered in identical $1 \mathrm{~kg}$ bags except for a three-digit code corresponding to the randomisation number, with up to $3 \mathrm{~kg} /$ month of salt substitute/salt available to each randomised participant to cover all cooking, pickling and other uses within the household. Correct packing and labelling of the study salt and the salt substitute was checked before the commencement of the study by testing the contents of a sample of randomised treatment packages. The participants were instructed to use the study salt for all food preparation throughout the study period. The post-randomisation use of the salt substitute/salt was determined at each visit by asking the study participants what proportion of the household salt used since the last visit, which was provided by the study, and recording a response of 'all', 'nearly all', 'half', 'less than half' or 'none'.

\section{Data collection}

Potentially eligible individuals attended a registration visit with the physician at the centre and consent was obtained, a baseline questionnaire was completed, a brief physical examination was performed and a blood sample was collected. Individuals willing to be long-term participants in the trial were entered into the active run-in period and instructed to return for a final eligibility check 4 weeks later. Eligible participants who attended the next scheduled visit were randomised. Food taste data were collected at registration, 1, 6 and 12 months post-randomisation visits. All data were collected on paper forms and then sent to Beijing and entered into a passwordprotected study website.

\section{Outcome assessments}

Three aspects of the taste of home-cooked foods and a standard salty soup ${ }^{(13)}$ were recorded using $100 \mathrm{~mm}$ visual analogue scales to document saltiness (from the left end marked 'not at all salty' to the right end marked 'very salty'), flavour (from the left end marked 'very weak' to the right end marked 'very strong') and overall acceptability (from the left end marked 'dislike extremely' to the right end marked 'like extremely'). For the assessment of home-cooked foods, the participants were asked to think about the usual taste of the food that they had eaten in the last month, and specifically about their opinion as to the saltiness, flavour and overall acceptability of their usual food. They were then asked to mark a horizontal visual analogue scale with a vertical line indicating how they think their usual food tasted in regard to each of the three aspects. The distance from the left end of the scale to the vertical line was measured using a ruler and recorded in millimetres. The results for saltiness, flavour and overall acceptability were collected on three separate scales on the same form at each visit. Assessment at follow-up visits was done in an identical manner, with participants blind to their previous results and their randomised allocation.

The same three components of taste were recorded at the same time points for a standard salty soup in an effort to define any change in the preferred level of saltiness over time. The soup provided was a commercially available dried soup mix product purchased in China. For each evaluation, one pack of the dried soup mix was added to a pan and mixed with $500 \mathrm{ml}$ of hot water $\left(60-70^{\circ} \mathrm{C}\right)$. The soup was then poured into small paper cups and when it had cooled to an acceptable temperature, the participants were asked to take a mouthful of the soup, to taste it and to swallow it. Their opinions as to the saltiness, flavour and overall 
acceptability of the soup mix were then recorded on the visual analogue scales. Aside from the provision of the soup, the data collection process was identical to that used for the assessment of home-cooked foods.

\section{Statistics}

Statistical analyses were carried out using the SAS for Windows, version 9.1. Mean levels (standard deviations) and proportions (standard errors) were calculated for baseline and follow-up characteristics. The analysis of treatment effects was by intention to treat with between-group comparisons for the outcomes of interest made using SAS Proc Mixed to fit mixed linear models for repeated-measures ANOVA. In the primary analyses, the registration visit and all of the follow-up measures for each of the outcomes were included in the mixed models with an overall estimate of effect, a CI and a $P$ value calculated. The secondary analysis on the overall estimate of effect was conducted with the weighting of each of the data points according to the lengths of the respective follow-up intervals to which the data point applied. The length of the follow-up interval was defined as the period between the present and previous assessment of taste; for example, the weight score of 6 was used for the final 12-month assessment, which was deemed to apply to the 6-month period between the 6-month and the 12-month visit. Possible changing effects of the randomised intervention over time were explored by fitting a visit by the treatment interaction term on the Proc Mixed model. A $P$ value less than 0.05 for the interaction term indicated evidence of a linear decreasing or increasing trend of the effect of the treatment over time. Since missing data were few (less than $5 \%$ for all analyses), no imputation procedures were undertaken. The comparison of adherence to the salt substitute/salt between the randomised groups was conducted using SAS Proc Genmod for generalised estimation equations ${ }^{(14)}$.

\section{Results}

There were 752 participants who registered for the study; 700 (93\%) who commenced run-in and $608(81 \%)$ who were randomised. The main reason for run-in participants failing to proceed to randomisation was reluctance to continue with the follow-up schedule ( $n$ 57). The participants who were randomised reported very good adherence to the study salt substitute/salt, with $99 \%$ indicating that they used the study salt substitute/salt for 'all' or 'nearly all' of their day-to-day food preparation with no difference between the randomised groups $(P=0 \cdot 40)$. Completeness of follow-up was also very high, with the final follow-up visit attended by $96 \%$ of the randomised participants and overall $98 \%$ of all post-randomisation visits completed as scheduled.

The mean age of the randomised participants was 60 years and $56 \%$ of them were females (Table 1). About two-thirds had a baseline history of vascular disease and one-sixth were included on the basis of a diagnosis of diabetes and an age of 55 years or older (Table 1).

Baseline mean ratings for saltiness, flavour and overall acceptability for the home-prepared food were directly comparable between the randomised groups (Table 1). The same
Table 1. Characteristics of the study participants by the randomised groups $^{*}$

\begin{tabular}{|c|c|c|}
\hline & $\begin{array}{c}\text { Salt } \\
\text { substitute } \\
(n 306)\end{array}$ & $\begin{array}{l}\text { Normal salt } \\
\quad(n \text { 302) }\end{array}$ \\
\hline Female & $166(52 \%)$ & $174(58 \%)$ \\
\hline Mean age, years (SD) & $59(10 \cdot 0)$ & $61(9 \cdot 7)$ \\
\hline \multicolumn{3}{|l|}{ Inclusion criteria } \\
\hline History of vascular disease & $189(62 \%)$ & $200(66 \%)$ \\
\hline $\begin{array}{l}\text { Systolic blood pressure } \\
\geq 160 \mathrm{mmHg}\end{array}$ & $173(57 \%)$ & $172(57 \%)$ \\
\hline $\begin{array}{l}\text { Treated diabetes and age } \\
>55 \text { years }\end{array}$ & $48(16 \%)$ & $57(19 \%)$ \\
\hline \multicolumn{3}{|l|}{ Smoking } \\
\hline Current smoker & $97(32 \%)$ & $85(28 \%)$ \\
\hline Past smoker & $45(15 \%)$ & $41(14 \%)$ \\
\hline Mean BMI, $\mathrm{kg} / \mathrm{m}^{2}$ (SD) & $26(3.6)$ & $25(3.9)$ \\
\hline $\begin{array}{l}\text { Mean blood pressure, } \\
\mathrm{mmHg}(\mathrm{SD})\end{array}$ & $159 / 93(25 / 14)$ & $159 / 93(26 / 14)$ \\
\hline \multicolumn{3}{|l|}{$\begin{array}{l}\text { Taste ratings for home-cooked } \\
\text { foods }\end{array}$} \\
\hline Saltiness, mm (SD) & $51(19)$ & $50(19)$ \\
\hline Flavour, mm (SD) & $62(18)$ & $61(18)$ \\
\hline Acceptability, mm (SD) & $69(15)$ & $67(17)$ \\
\hline \multicolumn{3}{|l|}{$\begin{array}{l}\text { Taste ratings for the standard } \\
\text { salty soup }\end{array}$} \\
\hline Saltiness, mm (SD) & $51(14)$ & $50(14)$ \\
\hline Flavour, mm (SD) & $67(17)$ & $64(14)$ \\
\hline Acceptability, mm (SD) & 69 (19) & $68(19)$ \\
\hline
\end{tabular}

${ }^{*}$ Number (per cent) unless otherwise indicated.

was also true for the ratings of the standard salty soup. During the follow-up, there were small variations in the ratings assigned to both home-prepared foods and the standard salty soup for each of the three parameters assessed, but the mean values in each randomised group fluctuated by just a few millimetres on the $100 \mathrm{~mm}$ scales used (Fig. 1). In general, about three-quarters of individual responses were within $10 \mathrm{~mm}$ above or below the mean response for each assessment. For the primary analyses, there were no detectable differences in the ratings between the randomised groups for saltiness, flavour or overall acceptability. This was true for both home-cooked foods and the standard soup (all $P>0.08$; Table 2). After weighting each of the data points according to the lengths of the respective follow-up intervals, the flavour of both home-cooked foods (mean difference $=-1.8 \mathrm{~mm}, \quad P=0.045$ ) and the standard salty soup (mean difference $=-1.9 \mathrm{~mm}, P=0.03$ ) was found to be weaker in the salt substitute group (Table 2).

Further exploration of the data showed that none of the individual baseline to follow-up differences in saltiness, flavour or overall acceptability between the randomised groups at separate time points were statistically significant for the evaluations of home-prepared foods (all $P>0 \cdot 05$ ). For the same assessments of the standard salty soup, there was a significant difference between the baseline and the 12-month follow-up only for the perception of flavour that was rated higher in those assigned to the normal salt group $(P=0.011$; Table 3$)$. An additional trend analysis suggested that the flavour of the standard salty soup was progressively more favourably perceived in the normal salt group over time $(P=0.03)$, although there was no corresponding finding for the flavour of homeprepared foods $(P=0 \cdot 2$; Fig. 1$)$. 
(a)

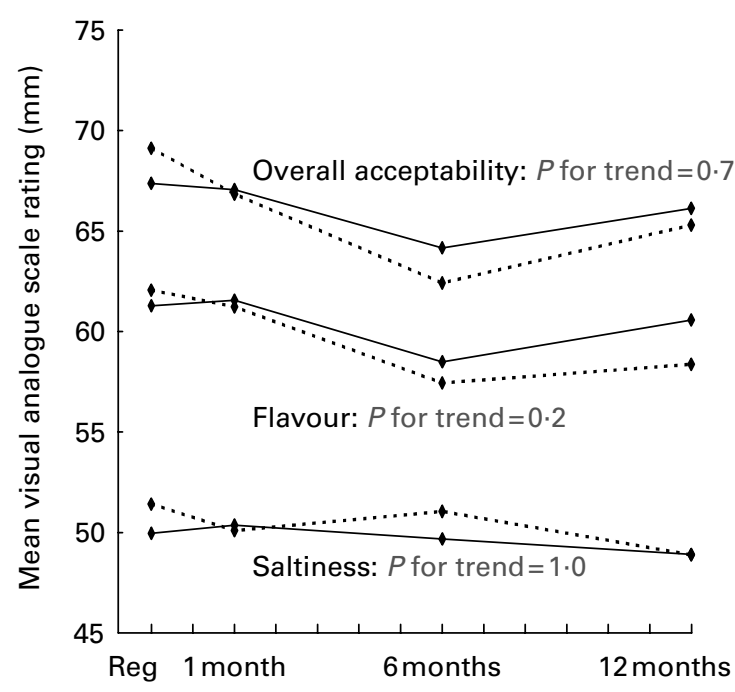

(b)

Fig. 1. Mean ratings of taste by the randomised groups and follow-up visits $(\mathrm{mm})$ for (a) usual home-prepared foods and (b) a standard salty soup: salt ( - ); salt substitute $(\ldots)$; Reg, registration. ' $P$ for trend' indicates the probability that there is a progressively increasing or decreasing difference between the randomised groups in the ratings over time.

\section{Discussion}

The China Salt Substitute Study has shown that replacing usual salt with a salt substitute in rural northern Chinese populations can bring about important reductions in blood pressure without producing a substantial adverse effect on the saltiness, flavour or overall acceptability of food. While the secondary weighted analyses did identify slightly impaired perception of flavour in the salt substitute group, the overall acceptability of food cooked with the salt substitute was not influenced and the vast majority of the participants continued to use the salt substitute throughout the study. This evidence of acceptability of the salt substitute is an important finding for public health because problems with taste are a widely cited reason for not using potassium-based salt substitutes that achieve blood pressure lowering through both the removal of sodium $^{(3)}$ and the addition of potassium ${ }^{(4)}$. These data provide significant support to the notion that advocating widespread use of this type of salt substitute for food preparation in northern China might be widely acceptable among the population.

There is previous evidence indicating that people are able to detect a difference when the taste of a usual food changes and that if the new stimulus added to the taste is unpleasant it will lead to disliking of the food ${ }^{(15)}$. Furthermore, this has been demonstrated even when the test of food taste and liking is done retrospectively ${ }^{(15)}$. It is therefore unlikely that the failure of the present study to detect substantial changes in the saltiness or overall acceptability of food reflects a failure of the study participants to identify or report differences that were present. Likewise, the large size of the study and substantial statistical power to detect

Table 2. Overall differences in taste ratings between the randomised groups for home-cooked foods and the standard salty soup $(\mathrm{mm})^{*}$

(Mean differences and 95\% confidence intervals)

\begin{tabular}{|c|c|c|c|c|c|c|}
\hline & \multicolumn{3}{|c|}{ Non-weighted method } & \multicolumn{3}{|c|}{ Weighted method $\dagger$} \\
\hline & Difference & $95 \% \mathrm{Cl}$ & $P$ & Difference & $95 \% \mathrm{Cl}$ & $P$ \\
\hline \multicolumn{7}{|c|}{ Home-cooked foods } \\
\hline Saltiness & 0.02 & $-1.39,1.44$ & 0.09 & 0.8 & $-0.76,2 \cdot 36$ & 0.80 \\
\hline Flavour & -1.4 & $-2 \cdot 95,0.16$ & 0.08 & $-1 \cdot 8$ & $-3.52,-0.05$ & 0.045 \\
\hline Acceptability & -1.3 & $-2.93,0.31$ & 0.97 & -1.6 & $-3 \cdot 34,0 \cdot 21$ & 0.09 \\
\hline \multicolumn{7}{|c|}{ Standard salty soup } \\
\hline Saltiness & -0.2 & $-1.57,1.17$ & 0.50 & 0.03 & $-1.48,1.54$ & 0.70 \\
\hline Flavour & -1.3 & $-2.76,0.25$ & 0.10 & -1.9 & $-3.57,-0.22$ & 0.027 \\
\hline Acceptability & -0.6 & $-2 \cdot 16,1.06$ & 0.78 & $-1 \cdot 1$ & $-1.53,-0.61$ & 0.23 \\
\hline
\end{tabular}

"Negative value means the rating of the salt substitute group was lower than the normal salt group.

†The weighted method was a post hoc analysis on the overall estimate of effect, which was conducted with the weighting of each of the data points according to the lengths of the respective follow-up intervals. 
Table 3. Baseline to follow-up differences in ratings of saltiness, flavour and acceptability between the randomised groups for home-cooked foods and the standard salty soup at each follow-up assessment $(\mathrm{mm})$

\begin{tabular}{|c|c|c|c|c|c|c|}
\hline & \multicolumn{3}{|c|}{ Home-cooked foods } & \multicolumn{3}{|c|}{ Standard salty soup } \\
\hline & 1 month & 6 months & 12 months & 1 month & 6 months & 12 months \\
\hline \multicolumn{7}{|l|}{ Saltiness } \\
\hline Difference* & -0.6 & $1 \cdot 13$ & -0.4 & -0.8 & 0.2 & $0 \cdot 10$ \\
\hline SE & $1 \cdot 1$ & 1.0 & $1 \cdot 1$ & 1.0 & $1 \cdot 0$ & $1 \cdot 1$ \\
\hline$P$ value & 0.6 & 0.3 & 0.7 & 0.4 & 0.9 & 0.95 \\
\hline \multicolumn{7}{|l|}{ Flavour } \\
\hline Difference & -0.5 & $-1 \cdot 3$ & $-2 \cdot 4$ & $0 \cdot 2$ & -1.0 & $-3 \cdot 0$ \\
\hline SE & $1 \cdot 1$ & $1 \cdot 1$ & $1 \cdot 2$ & $1 \cdot 1$ & $1 \cdot 1$ & $1 \cdot 1$ \\
\hline$P$ value & $0 \cdot 7$ & 0.2 & 0.05 & $0 \cdot 8$ & 0.4 & $0.011 \dagger$ \\
\hline \multicolumn{7}{|l|}{ Acceptability } \\
\hline Difference & -0.5 & $-2 \cdot 2$ & $-1 \cdot 2$ & 0.8 & -0.9 & -1.5 \\
\hline SE & $1 \cdot 1$ & $1 \cdot 1$ & $1 \cdot 2$ & $1 \cdot 2$ & $1 \cdot 1$ & $1 \cdot 3$ \\
\hline$P$ value & $0 \cdot 6$ & 0.05 & 0.3 & 0.5 & 0.4 & 0.2 \\
\hline
\end{tabular}

* Difference is the baseline to follow-up differences in ratings between the randomised groups (a negative value means the rating of the salt substitute group was lower than the normal salt group).

$\dagger P$ value $<0.05$

even small differences between groups makes it unlikely that the study failed to detect real differences in taste perception. There are, however, several possible reasons why changes in saltiness and acceptability of foods might have been minimal. First, reductions in saltiness of food consequent upon reduced sodium chloride content were likely offset by the addition of potassium chloride because potassium chloride also imparts a salty flavour ${ }^{(16)}$. Second, previous studies suggest that the reduction in saltiness caused by reducing sodium to the extent achievable with a salt substitute of this composition would have been only small to moderate ${ }^{(13,17)}$. Third, it is possible that other aspects of the food flavour such as umami (savoury taste) and odour were more important to taste than the saltiness component studied here. It is also known that any bitter taste caused by the potassium added at this level of substitution would have been small and would not impact greatly on the overall acceptability of salt substitutes ${ }^{(7)}$. Indeed, previous short-term studies of salt substitutes with this composition have shown that this combination of sodium, potassium and magnesium salts is well tolerated ${ }^{(7,18)}$, and this particular mix of sodium chloride, potassium chloride and magnesium sulphate has been widely marketed as a consequence.

The study provided some evidence of adverse changes in flavour, although this was apparent only in the secondary analyses. The magnitude of the observed effect was fairly small, being about $2 \mathrm{~mm}$ against a background spread of taste assessments in which three-quarters of the participants marked a response $10 \mathrm{~mm}$ above or below the mean. Perhaps more important from a public health perspective, any adverse effect on flavour caused by the salt substitute did not translate into changes in saltiness or overall acceptability of home-cooked foods.

The absence of any significant changes in the saltiness of the standard salty soup administered during the follow-up visits indicates that there was no significant resetting of individuals' taste sensitivity to salt. The individuals' preferred level of saltiness appears to be largely learned and to depend primarily upon the average level of salt consumed over the preceding few weeks. Foods with a saltiness that differs substantially from a person's usual level of exposure are likely to be less preferred whether they be more or less salty ${ }^{(19)}$. In the present study, the individuals assigned the salt substitute would have consumed on average about one-third less sodium chloride during the 12 months of the study. As such, it might have been anticipated that these individuals would have experienced a lowering in their preferred level of saltiness and that this would have been reflected in their reporting of the taste of the standard soup. The reason why there was no detectable resetting of the preferred level of saltiness is not clear. It is possible that the potassium chloride with which the sodium chloride was replaced in some way compensated for the reduction in sodium chloride, but in the absence of a clear scientific understanding of the basis for human salt taste it is difficult to be certain. There was evidence that as few as three exposures to an initially disliked salt level in a food can significantly increase the liking while the previously preferred salt level remains unchanged, indicating the possibility of a mere exposure explanation for the changes in liking ${ }^{(20)}$.

Key strengths of the present study are the randomised design, the large sample size and the rural community setting that helped minimise both systematic and random errors while ensuring that the result has direct applicability to large Chinese populations. One limitation of the pragmatic design was that it limited the extent of the sensory testing that could be done. So, while the study provides information about the acceptability of the salt substitute that is of direct clinical and public health applicability, it does not provide a good insight into the physiological reasons why the observed results were achieved.

In conclusion, these data show that the acceptability of salt substitution as a strategy for blood pressure control is unlikely to be importantly impacted by adverse effects of the salt substitute on food taste. If other practical aspects of making salt substitution feasible in rural China can be overcome, then it would seem that it is a strategy that would be widely acceptable and very effective. On the basis of the blood pressurelowering effects achieved, this strategy would appear to have the potential to reduce vascular disease in China by one-fifth $^{(8)}$ at a low cost and in a short time frame ${ }^{(2)}$. 


\section{Acknowledgements}

China Salt Substitute Study Collaborative Group: China, Tianjin, Cardiovascular Disease Research Institute, Hospital of People's Armed Police Forces: Yuming Li, Junxiang Liu, Donglin Song; Liaoning, Department of Clinical Epidemiology, The First Affiliated Hospital of China Medical University: Jingpu Shi, Hailong Wang, Yongqing Yang; Heilongjiang, Mudanjiang Centre for Disease Control and Prevention (The Institute for Medical Science): Jianmin Li, Yanling Zhang; Shanxi, The People's Hospital of Yu Xian: Dongshuang Guo, Shengyin Liang; Beijing, The Community Health Service Center of Yinghai, Daxing District: Jianhua Qi, Rujun Pan; The Community Health Service Center of Fengbo, Shunyi District: Bing Li, Zhigang Yang; Cardiovascular Institute and Fu Wai Hospital: Y. W., X. Y., L. Z., Bailing Chen, Beifan Zhou, Jihong Hu; The George Institute for International Health - China: Rong Han. Australia Sydney, The George Institute for International Health of the University of Sydney: N. L., B. N., F. B., Rachel Huxley, Kathy Jane, Suzanne Ryan; Ourimbah, School of Psychology, University of Newcastle: J. P. New Zealand, Auckland, Clinical Trial Research Unit of The University of Auckland: Anthony Rodgers, Carlene Lawes, Michelle Barlow, John Fa' atui, Amanda Milne, Colleen Ng, Simon Pink, Barry Gray.

The present study received financial support from The George Institute for International Health (Australia), The Clinical Trials Research Unit (New Zealand), The Capital Medical Science Development Fund (China) and the National Health and Medical Research Council of Australia (Grant ID: 358395). Personnel support was provided to N. L. as a scholarship from the National Health and Medical Research Council of Australia and to B. N. as a career development award from the National Heart Foundation of Australia. None of the collaborative group has any conflict to declare in regard to the present work.

The study was designed by B. N., Y. W. and L. Z. with input from J. P. Conduct of the project in China was under the control of Y. W., X. Y. and L. Z. with technical support from Australia and New Zealand provided by N. L. and B. N. Statistical analysis was done by F. B. and N. L. The initial draft of the paper was prepared by N. L., B. N. and J. P., with subsequent comments from all authors incorporated in the final report.

\section{References}

1. World Health Organization (2002) World Health Report 2002; Reducing Risk, Promoting Healthy Life. Geneva: World Health Organization.

2. Murray CJ, Lauer JA, Hutubessy R, Niessen L, Tomijima N, Rodgers A, Lawes CM \& Evans DB (2003) Reducing the risk of cardiovascular disease: effectiveness and costs of interventions to reduce systolic blood pressure and cholesterol - a global and regional analysis. Lancet 361, 717-725.
3. Cutler JA, Follmann D \& Allender PS (1997) Randomized trials of sodium reduction: an overview. Am J Clin Nutr $\mathbf{6 5}$, 643S-651S

4. Whelton PK, He J, Cutler JA, Brancati FL, Appel LJ, Follmann D \& Klag MJ (1997) Effects of oral potassium on blood pressure - meta-analysis of randomized controlled clinical trials. J Am Med Assoc 277, 1624-1632.

5. Tian H-G, Guo Z-Y, Hu G, Yu S-J, Sun W, Pietinen P \& Nissinen A (1995) Changes in sodium intake and blood pressure in a community-based intervention project in China. $J$ Hum Hypertens 9, 959-968.

6. Nan Y, Tian H-G, Shao R-C, Hu G, Dong Q-N, Pietinen P \& Nissinen A (1995) Assessment of sodium and potassium in processed foods in an urban area in China. Eur J Clin Nutr 49, 299-306

7. Karppanen H, Tanskanen A, Tuomilehto J, Puska P, Vuori J, Jantti V \& Seppanen ML (1984) Safety and effects of potassium- and magnesium-containing low sodium salt mixtures. J Cardiovasc Pharmacol 6, Suppl. 1, S236-S243.

8. The China Salt Substitute Study Collaborative Group (2007) Salt substitution: a low-cost strategy for blood pressure control among rural Chinese. A randomized, controlled trial. J Hypertens 25, 2011-2018.

9. Eertmans A, Baeyens F \& Van den Bergh O (2001) Food likes and their relative importance in human eating behavior: review and preliminary suggestions for health promotion. Health Educ Res 16, 443-456.

10. Drewnowski A \& Gomez-Carneros C (2000) Bitter taste, phytonutrients, and the consumer: a review. Am J Clin Nutr 72, 1424-1435.

11. Bartoshuk LM, Rifkin B, Marks LE \& Hooper JE (1988) Bitterness of $\mathrm{KCl}$ and benzoate: related to genetic status for sensitivity to PTC/PROP. Chem Senses 13, 517-528.

12. Bertino M, Beauchamp G \& Engelman K (1982) Long-term reduction in dietary sodium alters the taste of salt. $\mathrm{Am} \mathrm{J} \mathrm{Clin}$ Nutr 36, 1134-1140.

13. Girgis S, Neal B, Prescott J, Prendergast J, Dumbrell S, Turner C \& Woodward M (2003) A one-quarter reduction in the salt content of bread can be made without detection. Eur J Clin Nutr 57, 616-620.

14. Woodward M (2005) Epidemiology: Study Design and Data Analysis, 2nd ed. London: Chapman and Hall/CRC Press.

15. Koster MA, Prescott J \& Koster EP (2004) Incidental learning and memory for three basic tastes in food. Chem Senses 29, $441-453$

16. Rosett T, Wu Z, Schmidt S, Ennis D \& Klein B (1995) KCI, $\mathrm{CaCI}, \mathrm{Na}^{+}$binding, and salt taste of gum systems. J Food Sci 60, $849-853$.

17. Adams SO, Maller O \& Cardello AV (1995) Consumer acceptance of foods lower in sodium. J Am Diet Assoc 95, 447-453.

18. Gelejinske J (1994) Reduction in blood pressure with a low sodium, high potassium, high magnesium salt in older subjects with mild to moderate hypertension. Br Med J 309, 456-459.

19. Beauchamp GK \& Engelman K (1991) High salt intake. Sensory and behavioral factors. Hypertension 17, Suppl. 1, I176-I181.

20. Prescott J \& Khu B (1995) Changes in preference for saltiness within soup as a function of exposure. Appetite 24, 302. 